\title{
A cárie precoce da infância: uma atualização
}

Elisa Laranjo, ${ }^{1}$ Sofia Baptista, ${ }^{2}$ Ana Alves Norton, ${ }^{3}$ Ana Paula Macedo, ${ }^{3}$ Casimiro de Andrade, ${ }^{3}$ Cristina Areias $^{3}$

\section{RESUMO}

Objetivos: Fornecer informações para auxiliar o médico especialista em medicina geral e familiar na identificação dos fatores de risco associados à cárie precoce da infância (CPI), uma das doenças crónicas mais prevalentes da infância, salientando os primeiros sinais da doença e as complicações inerentes.

Fontes de dados: As informações foram selecionadas a partir de quinze artigos publicados nos últimos anos, entre 2006 e 2017, em inglês e português, nas bases de dados PubMed, Scopus e EBSCOhost, livros técnicos e publicações de consenso de organismos internacionais.

Conclusões: Os médicos de família desempenham um importante papel na prevenção da CPI, sendo fundamental a formação e a atualização em saúde oral.

Palavras-chave: Cárie precoce da infância; Prevenção; Crianças

\section{INTRODUÇÃO}

A cárie dentária está presente em todo o mundo e é responsável pela destruição e perda dos dentes. É uma das doenças mais prevalentes da infância e exerce forte impacto no bem-estar individual e social da criança. É uma patologia infeciosa comum, crónica e transmissível, resultante da atividade de bactérias específicas que aderem à superfície dentária, principalmente Streptococcus mutans (SM), que metabolizam açúcares para a produção de ácido que, ao longo do tempo, desmineraliza o esmalte. ${ }^{1}$

A cárie em crianças de idade pré-escolar tem vindo a ser referida como early childhood caries, traduzida para português como cárie precoce da infância (CPI), também designada anteriormente como «cárie de biberão».

A CPI caracteriza-se pela presença de um ou mais dentes decíduos com lesão de cárie (cavitada ou não), restaurados/obturados ou perdidos devido a cárie, em crianças menores de seis anos de idade. ${ }^{1-2}$

1. Médica Dentista. Especialização em Odontopediatria. Centro de Saúde de Ponta Delgada, Açores.

2. Médica Dentista. Especialização em Odontopediatria.

3. Médico Dentista. Professor de Odontopediatria. Faculdade de Medicina Dentária, da Universidade do Porto.
Nas crianças com idade inferior a três anos qualquer sinal de lesão de cárie em superfícies dentárias lisas é indicativo de «cárie precoce de infância grave» (CPI grave). Igualmente se considera CPI grave nas crianças entre os três/cinco anos com a presença de um ou mais dentes decíduos maxilares anteriores cariados, perdidos (por cárie) ou restaurados/obturados; um valor de dentes cariados perdidos e obturados (cpod) maior ou igual a quatro aos três anos; um cpod maior ou igual a cinco aos quatro anos e um cpod maior ou igual a seis aos cinco anos. ${ }^{2}$

A American Academy of Pediatric Dentistry reconheceu a CPI como um problema significativo de saúde pública. $^{3}$

A família tem influência na definição de comportamentos de saúde oral e tem um papel fundamental no desenvolvimento dos hábitos e dos conhecimentos da criança.

Os dados epidemiológicos sobre a CPI são escassos, mas indicam claramente que a CPI é altamente prevalente. $^{4-5}$

\section{Etiologia da CPI}

Sendo a cárie dentária uma doença infeciosa transmissível, o SM e espécies de Lactobacillus são incluídos nos marcadores de risco microbiano para CPI. ${ }^{6} \mathrm{~A}$ transmissão de SM é provavelmente vertical a partir do cui- 
dador para a criança, através do contacto salivar, sendo dependente da frequência e quantidade de exposição. Assim, as crianças cujas mães têm altos níveis de SM estão em maior risco de contrair a bactéria. ${ }^{7}$ A transmissão horizontal, entre outros membros da família ou das outras crianças nas creches, por exemplo, também ocorre. $^{7}$

Os dentes recém erupcionados, devido ao seu esmalte imaturo, e dentes com hipoplasia do esmalte podem estar em maior risco de desenvolver cárie.

\section{Prevenção da CPI \\ Higiene oral e flúor}

As práticas atuais, apoiadas pela Direção-Geral da Saúde, recomendam a escovagem dentária duas vezes por dia com pasta dentífrica fluoretada (1.000-1.500 ppm de flúor) para todas as crianças de comunidades com águas otimamente fluoretadas (como nas Regiões Autónomas) e deficientes em flúor. ${ }^{8}$

Para crianças com menos de três anos de idade deve ser utilizada uma quantidade de pasta fluoretada do tamanho de um «grão de arroz». Dos três aos seis anos de idade é apropriada uma quantidade do tamanho de um «grão de ervilha»" ${ }^{8-9}$ (Figura 3 e Quadro I).

Os pais devem colocar a pasta dentífrica numa escova macia, de tamanho adequado à idade e executar ou auxiliar a escovagem dentária. Para maximizar o efeito benéfico do flúor da pasta dentífrica, o enxaguamento após a escovagem deve ser reduzido ao mínimo ou completamente eliminado ${ }^{10}$ (Quadro I).

\section{Alimentação}

Um fator de risco associado à etiologia microbiana é o consumo frequente de açúcar. As práticas dietéticas cariogénicas parecem ser estabelecidas por volta dos doze meses de idade e são mantidas durante toda a infância. ${ }^{11-12}$ Apesar de a amamentação natural não estar consistentemente implicada com a CPI, ${ }^{13}$ a alimentação com leite materno em combinação com outros hidratos de carbono demonstrou in vitro ser altamente cariogénica. ${ }^{14}$

O consumo frequente de snacks e bebidas açucaradas entre as refeições aumenta o risco de cárie devido ao contacto prolongado entre os açúcares dos alimentos ou líquidos consumidos e bactérias cariogénicas sobre os dentes suscetíveis. ${ }^{15}$

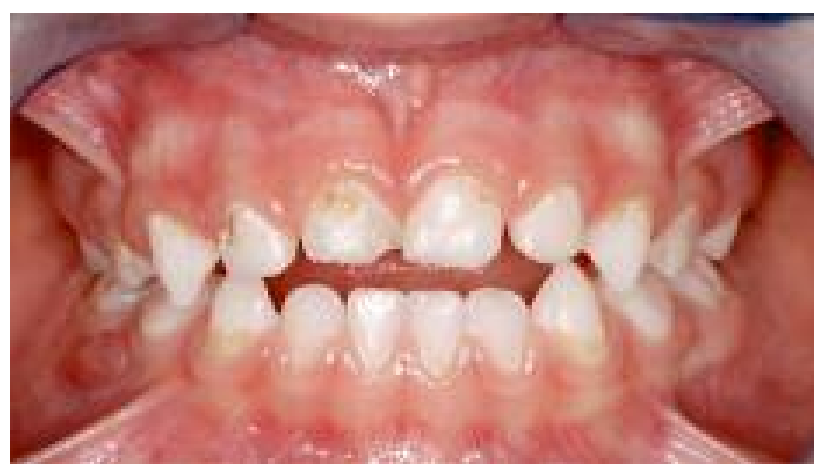

Figura 1. Lesões iniciais de cárie nos dentes anteriores em criança de três anos (assinale-se a fístula na zona dos molares inferiores direitos).

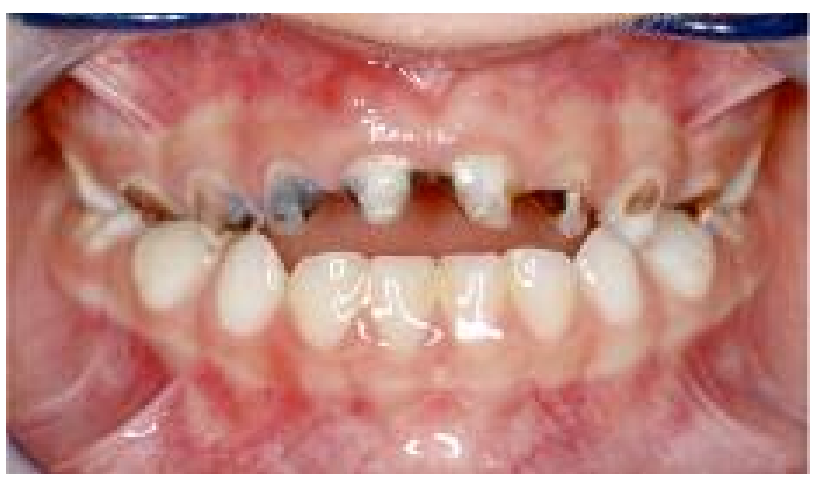

Figura 2. Lesões graves de cárie em criança de quatro anos.

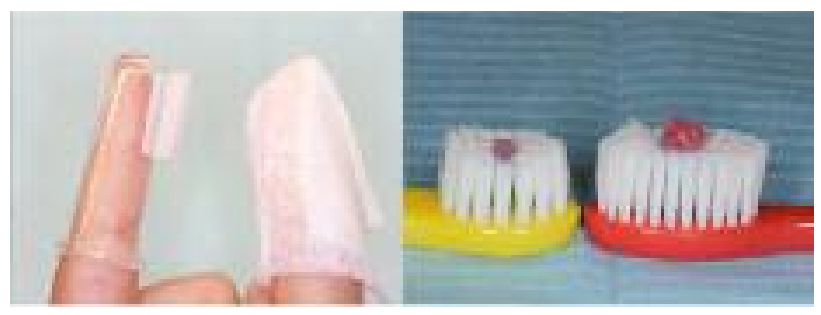

Figura 3. Dedeira e gaze para auxílio na higiene oral e quantidade de dentífrico fluoretado respeitando a faixa etária (escova amarela com uma quantidade de «um grão de arroz» e escova vermelha com uma quantidade de «uma ervilha».

\section{Consequências da CPI, segundo a American Academy} of Pediatric Dentistry (AAPD) ${ }^{3}$

As consequências da CPI incluem:

- Um maior risco de novas lesões de cárie em ambas as dentições

- Mais hospitalizações e consultas de urgência

- Custos aumentados de tratamento 


\begin{tabular}{|c|c|c|c|c|}
\hline Recomendações & $\begin{array}{l}\text { Frequência da } \\
\text { escovagem }\end{array}$ & $\begin{array}{l}\text { Material usado } \\
\text { na escovagem }\end{array}$ & $\begin{array}{l}\text { Execução da } \\
\text { escovagem }\end{array}$ & $\begin{array}{l}\text { Dentífrico } \\
\text { fluoretado }\end{array}$ \\
\hline $\begin{array}{l}\text { Antes da erupção } \\
\text { do } 1^{\circ} \text { dente }\end{array}$ & $\begin{array}{l}\text { 2x dia } \\
\text { (higiene das } \\
\text { mucosas orais) }\end{array}$ & $\begin{array}{l}\text { Gaze } \\
\text { Dedeira }\end{array}$ & Pais & - \\
\hline $\begin{array}{l}\text { A partir da erupção do } \\
1^{\circ} \text { dente }-3 \text { anos }\end{array}$ & $\begin{array}{l}\text { 2x dia } \\
\text { (sendo obrigatória } \\
\text { 1x à noite) }\end{array}$ & $\begin{array}{l}\text { Macia e de } \\
\text { tamanho } \\
\text { adequado à boca } \\
\text { da criança }\end{array}$ & Pais e criança & $\begin{array}{l}\text { Com 1.000-1.500 ppm } \\
\text { de flúor } \\
\text { «um grão de arroz» }\end{array}$ \\
\hline 3-6 anos & $\begin{array}{l}\text { 2x dia } \\
\text { (sendo obrigatória } \\
\text { 1x à noite) }\end{array}$ & $\begin{array}{l}\text { Macia e de } \\
\text { tamanho } \\
\text { adequado à boca } \\
\text { da criança }\end{array}$ & Pais e criança & $\begin{array}{l}\text { Com 1.000-1.500 ppm } \\
\text { de flúor } \\
\text { «uma ervilha» }\end{array}$ \\
\hline
\end{tabular}

- Risco de atraso no crescimento físico e desenvolvimento

- Perda de dias de escola e aumento de dias com atividade restrita

- Diminuição da capacidade de aprender

- Diminuída qualidade de vida

\section{Recomendações da AAPD e Departamento de} Odontopediatria da Faculdade de Medicina Dentária da Universidade do Porto ${ }^{3}$

1. Redução dos níveis de SM dos pais e irmãos da criança para diminuir a transmissão de bactérias cariogénicas. As grávidas seguidas no Serviço Nacional de Saúde e as crianças e jovens em meio escolar da rede pública e instituições particulares de solidariedade social dos sete aos quinze anos beneficiam do Programa Nacional de Promoção de Saúde Oral (vulgo cheque-dentista).

2. Minimizar atividades de partilha de saliva (e.g., partilha de talheres entre crianças, provar a comida com o mesmo talher com que se vai dar à criança, beijos na boca, limpar a chupeta com a boca antes de dar à criança) para diminuir a transmissão de bactérias cariogénicas.

3. Implementação de medidas de higiene oral, o mais tardar, no momento da erupção do primeiro dente temporário, usando uma escova macia de tamanho apropriado para a idade e dentífrico fluoretado.
4. Aplicações profissionais de verniz fluoretado às crianças em risco para a CPI.

5. Estabelecer o acompanhamento da criança (Dental Home) no prazo de seis meses a contar da erupção do primeiro dente ou, o mais tardar, aos doze meses de idade, para se realizar uma avaliação de risco de cárie e fornecer educação aos pais, incluindo orientações para a prevenção das doenças orais.

6. Evitar o consumo frequente de líquidos e/ou alimentos sólidos que contenham açúcar. Em particular:

- Bebidas contendo açúcar (e.g., sumos, refrigerantes, chá adoçado, leite com adição de açúcar) em biberões ou em copos com bico deve ser evitado.

- As crianças não devem ser adormecidas com biberão com leite ou líquidos que contenham açúcar.

- Após a erupção do primeiro dente temporário, outros hidratos de carbono dietéticos devem ser introduzidos para além da amamentação natural.

- Os pais devem encorajar os seus filhos a beber de um copo perto do primeiro aniversário. As crianças devem ser desmamadas do biberão entre os doze a dezoito meses de idade.

7. Assegurar que todos os bebés e crianças tenham acesso a rastreios dentários, aconselhamento e tratamentos preventivos. 


\section{REFERÊNCIAS BIBLIOGRÁFICAS}

1. Drury TF, Horowitz AM, Ismail Al, Maertens MP, Rozier RG, Selwitz RH. Diagnosing and reporting early childhood caries for research purposes. J Public Health Dent. 1999;59(3):192-7.

2. American Academy of Pediatric Dentistry. Policy on early childhood caries: classifications, consequences, and preventive strategies. Ref Manual. 2016;39(6):59-61.

3. American Academy of Pediatric Dentistry. Symposium on the prevention of oral disease in children and adolescents, November 11-12, 2005. Pediatr Dent 2006;28(2):96-198.

4. Vasconcelos NP, Melo P, Gavinha S. Estudo dos factores etiológicos das cáries precoces da infância numa população de risco. Rev Port Estomatol Cir Maxilofac. 2004;45(2):69-77.

5. Mendes S, Bernardo M. Cárie precoce da infância nas crianças em idade pré-escolar do distrito de Lisboa (critérios International Caries Detection and Assessment System II) [Early childhood caries in pre-school children of Lisbon (International Caries Detection and Assessment System II criteria)]. Rev Port Estomatol Med Dent Cir Maxilofac. 2015;56(3):156-65. Portuguese

6. Kanasi E, Johansson I, Lu SC, Kressin NR, Nunn ME, Kent R Jr, et al. Microbial risk markers for childhood caries in pediatricians' offices. J Dent Res. 2010;89(4):378-83.

7. Berkowitz RJ. Mutans streptococci: acquisition and transmission. Pediatr Dent. 2006;28(2):106-9.

8. Direção-Geral da Saúde. Programa nacional de promoção da saúde oral: circular normativa n. ${ }^{\circ}$ 1/DSE, de 18/01/05. Lisboa: DGS; 2005.

9. American Dental Association Council on Scientific Affairs. Fluoride toothpaste use for young children. J Am Dent Assoc. 2014;145(2):190-1.
10. Sjögren K, Birkhed D. Factors related to fluoride retention after toothbrushing and possible connection to caries activity. Caries Res. 1993;27(6):474-7.

11. Douglass JM. Response to Tinanoff and Palmer: Dietary determinants of dental caries and dietary recommendations for preschool children. J Public Health Dent. 2000;60(3):207-9.

12. Kranz S, Smiciklas-Wright $H$, Francis LA. Diet quality, added sugar, and dietary fiber intakes in American preschoolers. Pediatr Dent. 2006;28(2):164-71.

13. Reisine S, Douglass JM. Psychosocial and behavioral issues in early childhood caries. Community Dent Oral Epidemiol. 1998;26(1 Suppl):32-44.

14. Erickson PR, Mazhari E. Investigation of the role of human breast milk in caries development. Pediatr Dent. 1999;21(2):86-90.

15. Tinanoff N, Palmer CA. Dietary determinants of dental caries and dietary recommendations for preschool children. J Public Health Dent. 2000;60(3):197-206.

\section{CONFLITO DE INTERESSES}

Os autores declaram não ter conflitos de interesses.

\author{
ENDEREÇO PARA CORRESPONDÊNCIA \\ Elisa Laranjo \\ E-mail: elisalaranjo@gmail.com
}

Recebido em 16-06-2016

Aceite para publicação em 19-12-2017

\section{ABSTRACT}

\section{EARLY CHILDHOOD CARIES: AN UPDATE}

Background: Childhood caries is one of the most common chronic childhood diseases.

Aim: To identify risk factors for early childhood caries, highlighting their early clinical presentation and the inherent complications.

Data source: Information was retrieved from fifteen articles published between 2006 and 2017, in English and Portuguese languages, in PubMed, Scopus and EBSCOhost, complemented by manual search in technical books and guidelines of international societies.

Conclusions: Primary care is crucial for effective prevention of ECC. Family physicians are the better placed providers to do it. Updated education on oral health is mandatory.

Keywords: Early childhood caries; Prevention; Children 